\title{
Ephaptic Coupling of Myelinated Nerve Fibers
}

\author{
S. Binczak ${ }^{\mathrm{a}}$, J.C. Eilbeck ${ }^{\mathrm{b}}$ and A.C. Scott ${ }^{\mathrm{c}}$ \\ a) Laboratoire LE2I, Electronique, Informatique et Image \\ Universite de Bourgogne \\ BP 40021011 Dijon, France \\ b) Department of Mathematics \\ Heriot-Watt University \\ Edinburgh EH14 4AS, Scotland \\ c) Department of Mathematical Modelling \\ Technical University of Denmark \\ DK-2800 Lyngby, Denmark
}

May 23, 2000

\begin{abstract}
Numerical predictions of a simple myelinated nerve fiber model are compared with theoretical results in the continuum and discrete limits, clarifying the nature of the conduction process on an isolated nerve axon. Since myelinated nerve fibers are often arranged in bundles, this model is used to study ephaptic (nonsynaptic) interactions between impulses on parallel fibers, which may play a functional role in neural processing.
\end{abstract}

\section{Introduction}

Following the quantitative formulation of nerve impulse dynamics for the giant axon of the squid by Hodgkin and Huxley in 1952 [17], many of the analytic studies have focused on smooth nerve fibers which are described by nonlinear partial differential equations (nonlinear reaction diffusion equations). Although this picture is appropriate for the squid axon, many nerve fibers are discrete, periodic structures, comprising active nodes separated by sections of nerve that 
are insulated by myelin. In such myelinated nerves, as Rushton showed in 1951 [38], the wave of activity jumps from one node to the next and can be described by nonlinear difference-differential equations.

Called "saltatory" conduction by the electrophysiologists, impulse propagation on myelinated nerves is qualitatively similar to a row of falling dominos and to the signal fires of coastal warning systems during the Middle Ages. Myelinated nerve structures are useful because they allow an increase in the speed of a nerve impulse while decreasing the diameter of the nerve fiber; thus the motor nerves of vertebrates - which are about the same diameter as a squid giant axon - comprise several hundred individual fibers, each serving as an independent signaling channel [46]. As an added advantage, myelinated nerves expend much less energy in transmitting an impulse.

In general, neurons talk to each other through chemical junctions called "synapses," across which excitatory or inhibitory substances are released by incoming nerve impulses, thereby modifying the dendritic membranes of subsequent neurons, but there are other possibilities. In this paper, we consider the dynamics of impulses on myelinated nerve fibers that are parallel and relatively close together (as in vertebrate motor nerves) so the electrodynamics on one fiber can influence those on others and vice versa. Such parallel fiber interactions have been known to electrophysiologists since the 1940s [2, 22], and are termed ephaptic from a Greek word meaning "to touch" [21].

First studied analytically and numerically in the 1960s [3, 13, 14, 15, 19, $20,26,27,28,33,34,36,37,39]$, saltatory conduction on myelinated nerve models introduces two qualitatively important features. One of these is the above mentioned increase in speed of conduction, and another is the possibility of failure when the distance (or electrical resistance) between the active nodes becomes too large. We show here that both of these phenomena are influenced by ephaptic coupling.

A simple model for computing the leading edge propagation of an impulse is developed in Section 2 of this paper, clarifying the nature of conduction on a real nerve and setting the stage for an exploration of the effects of ephaptic interactions. In section 3, we discuss the phenomena of impulse synchronization due to ephaptic interactions in the saltatory and continuum limits from theoretical perspectives, and in Section 4 numerical results on three effects of ephaptic coupling are presented: (i) the formation of synchronized pulses on neighboring fibers, (ii) the ratio of the conduction velocity of such coupled impulses to the corresponding velocity of an uncoupled (free) impulse, and (iii) changes in the onset of failure.

\section{$2 \quad$ An isolated myelinated fiber}

Our aim in this section is to develop a simple yet physically reasonable model for impulse propagation on a myelinated nerve fiber, and demonstrate its ability to 


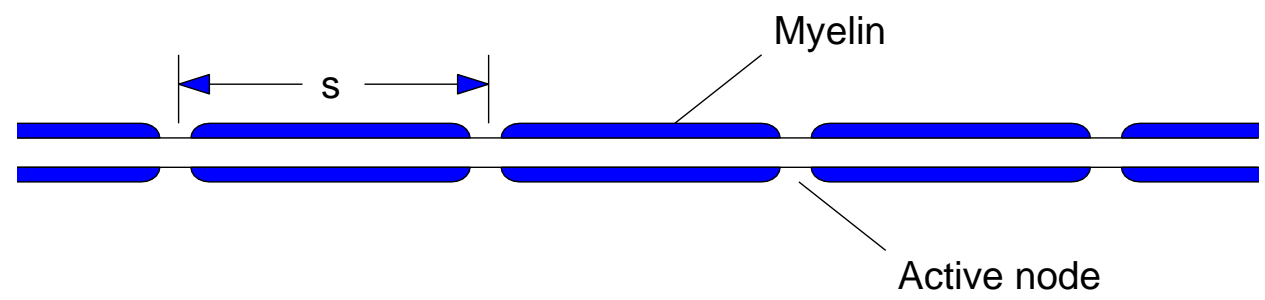

Figure 1: A single myelinated nerve fiber. (not to scale.)

describe theoretical results and experimental observations. (See reference [44] for an up-to-date information on many aspects of real myelinated axons.)

\subsection{An electric circuit model}

In Figure 1 is sketched a single myelinated nerve fiber, showing active nodes which are separated by regions of the fiber that are insulated by myelin. This structure can be modeled by the following system of difference-differential equations [31].

$$
V_{n}-V_{n+1}=\left(R_{\mathrm{i}}+R_{\mathrm{o}}\right) I_{n}
$$

and

$$
I_{n-1}-I_{n}=C \frac{d V_{n}}{d t}+I_{\text {ion }, n} .
$$

The index $n$ indicates successive active nodes, each of which is characterized by a transverse (inside to outside) voltage across the membrane $\left(V_{n}\right)$. A second dynamic variable is the current $\left(I_{n}\right)$ flowing longitudinally through the fiber from node $n$ to node $n+1$. Thus Equation (1) is merely Ohm's law, relating the voltage difference between two adjacent nodes of the current flowing between them times the sum of the inside and outside resistances: $R_{\mathrm{i}}$ and $R_{\mathrm{O}}$.

Equation (2) says that the current flowing into the $n$th node from the $(n-$ 1 ) th node $\left(I_{n-1}\right)$ minus the current flowing out of it to the $(n+1)$ th node $\left(I_{n}\right)$ is equal to the following two components of transverse (inside to outside) current leaving the node: (i) the capacitive current, $C d V_{n} / d t$, and (ii) the ionic current, $I_{\text {ion, } n}$, comprising both sodium and potassium components [17].

The time delay for the onset of sodium ion permeability is rather short (in a frog's myelinated motor axon it is less than 0.1 millisecond), whereas the time delay for the onset of potassium ion permeability is several milliseconds [8]. Thus it is appropriate to assume that the sodium ion current begins without 
delay, and the potassium permeability remains equal to its resting value over the leading edge of the impulse. From the Hodgkin-Huxley formulation, these assumptions imply that the total ionic current is represented by a nonlinear conductivity [17]. ${ }^{1}$ To maintain contact with several analytic results that are available for nonmyelinated nerves $[40,41]$, we further assume that this ionic current is given by the cubic expression

$$
I_{\text {ion }, n}=\left(\frac{G}{V_{\mathrm{b}}\left(V_{\mathrm{b}}-V_{\mathrm{a}}\right)}\right) V_{n}\left(V_{n}-V_{\mathrm{a}}\right)\left(V_{n}-V_{\mathrm{b}}\right) .
$$

In this expression, the resting potential of the active membrane is zero, and the parameters are defined as follows. (i) The threshold voltage at which sodium current begins to flow into an active node is $V_{\mathrm{a}}$. (ii) The Nernst (diffusion) potential at which total (primarily sodium) ion current returns to zero is $V_{\mathrm{b}}$. (iii) The total (primarily sodium) ionic conductance near $V_{\mathrm{b}}$ is $G$.

Next we consider how the parameters of this model are related to the experimental description of a real myelinated nerve fiber. To this end, we turn to the following measurements on a single axon (with a diameter of 14 microns) of a frog's motor nerve [18, 8, 43]: (i) Distance between nodes $=2 \mathrm{~mm}$. (ii) Internal resistance/length $=140-145 \mathrm{megohm} / \mathrm{cm}$. (iii) Capacity of myelin $/$ length $=10$ to 16 picofarad $/ \mathrm{cm}$. (iv) Capacity of active node $=0.6$ to 1.5 picofarad.

From this data

- $R_{\mathrm{i}}=28 \pm 1$ megohm, and

- $C=3.7 \pm 1$ picofarad,

where it will be noted that the distributed capacitance of the internodal myelin sheath has been lumped together with nodal membrane capacitance. For an isolated nerve fiber in an experimental situation, the cross-section for external current flow is much greater than that for internal resistance; thus

- $R_{\mathrm{O}} \ll R_{\mathrm{i}}$,

however larger values of $R_{\mathrm{o}}$ are to be expected in nerve bundles, where many individual fibers are situated together [44].

Cole has reported the maximum sodium conductance of a frog node as [8]

- $G=0.57$ micromhos,

and the Nernst potential for sodium ions as

- $V_{\mathrm{b}}=122$ millivolts.

Also the threshold potential for a typical nerve membrane is about [40]

\footnotetext{
${ }^{1}$ In the Hodgkin-Huxley notation, the sodium turn-on variable $m=m_{0}\left(V_{n}\right)$, and the sodium turn-off and potassium turn-on variables are respectively $h=h_{0}(0)$ and $n=n_{0}(0)$.
} 
- $V_{\mathrm{a}} \sim 25$ millivolts.

Potassium ion current carries positive charge out of an active node; thus it is a recovery variable. An exact expression for this current was presented by Hodgkin and Huxley [17] and a simple representation was proposed by FitzHugh [12], but here we note that the time delay for the onset of potassium current is about 4 milliseconds [8]. Since the length of the impulse is about equal to this time delay times its speed ( 23 millimeters/millisecond), we expect the trailing edge of an impulse to lag behind its leading edge by about 9 centimeters or 45 nodes. Thus - as noted above - it is reasonable to neglect effects of the trailing edge of the impulse on its leading edge dynamics. We shall employ this approximation throughout the present paper by assuming that the potassium ion permeability remains equal to its resting value.

For the experimental parameters that are listed above, we can measure voltages in units of the Nernst potential $\left(V_{\mathrm{b}}\right)$ to obtain the dimensionless voltage variables

$$
v_{n} \equiv V_{n} / V_{\mathrm{b}} .
$$

Then Equations (1) and (2) become the discrete reaction diffusion system

$$
R C \frac{d v_{n}}{d t}=\left(v_{n+1}-2 v_{n}+v_{n-1}\right)-\left(\frac{R G}{1-a}\right) v_{n}\left(v_{n}-a\right)\left(v_{n}-1\right),
$$

where

$$
a \equiv V_{\mathrm{a}} / V_{\mathrm{b}}, \text { and } R \equiv\left(R_{\mathrm{i}}+R_{\mathrm{o}}\right)
$$

At this point, we wish to normalize the model in a manner that: (i) allows the internode spacing to be an independent parameter, and (ii) maintains contact with standard notations in studies of discrete nonlinear diffusion by applied mathematicians. To these ends we take $s$ to be a variable internodal distance and define a discreteness parameter

$$
D \equiv\left(\frac{2 \mathrm{~mm}}{s}\right)=\frac{R_{\mathrm{f}}}{R}
$$

where it is intended throughout this paper that

$$
R_{\mathrm{f}}=28 \text { megohms }
$$

which is the internode resistance of a frog nerve. In other words, $1 / D$ is the spacing between nodes in units of $2 \mathrm{~mm}$, so $D=1$ implies the discreteness of a standard frog nerve.

In this formulation, the dynamic equation becomes 


$$
D\left(v_{n+1}-2 v_{n}+v_{n-1}\right)=R_{\mathrm{f}} C \frac{d v_{n}}{d t}+\beta v_{n}\left(v_{n}-a\right)\left(v_{n}-1\right),
$$

where

$$
\beta \equiv \frac{R_{\mathrm{f}} G}{1-a} .
$$

Although the experimental values of the parameters upon which Equation (5) is based are only approximately determined, we believe that they provide reasonable estimates for numerical studies of myelinated motor nerves of a frog.

\subsection{Impulse speed and failure}

In Equation (5), we have obtained a discrete reaction diffusion system, modeling a myelinated nerve. Here we briefly present some numerical calculations of impulse speeds and compare them with certain theoretical results $[1,10,28,36$, 37].

Broadly speaking, the nature of the wave propagation on a discrete nerve model can be characterized by looking at the relative change in voltage between two adjacent nodes. If this relative change everywhere satisfies the inequality $\left|\left(v_{n+1}-v_{n}\right) / v_{n}\right| \ll 1$, then the voltages and currents are smooth functions of distance and the system can be described by partial differential equations: the corresponding continuum system. If, on the other hand, $\max \mid\left(v_{n+1}-\right.$ $\left.\left.v_{n}\right) / v_{n}\right) \mid \gg 1$, then the conduction process is saltatory, jumping from one active node to the next in a discontinuous manner. We shall refer to these two cases as the continuum limit and saltatory limit respectively.

\section{Continuum limit}

If the internodal spacing $s$ is small enough so that the continuum limit is reached, then Equation (5) can be written as the partial differential equation

$$
s^{2} D \frac{\partial^{2} v}{\partial x^{2}}-R_{\mathrm{f}} C \frac{\partial v}{\partial t}=\beta v(v-a)(v-1),
$$

where we have let

$$
n s \rightarrow x
$$

Formulated in 1938 as a model for flame front propagation, Equation (6) has been extensively studied as a simple nerve model since the early 1960s [41]. In particular, if we measure time in units of $R_{\mathrm{f}} C / \beta$ and distance in units of $s \sqrt{D / \beta}$, then a traveling wave front (the leading edge of the impulse) was shown by Zel'dovich and Frank-Kamenetsky to have shape [47] 


$$
v(x, t)=\frac{1}{1+\exp [(x-u t) / \sqrt{2}]}
$$

and speed

$$
u=(1-2 a) / \sqrt{2} .
$$

From Equation (6), a condition for the continuum approximation to hold is

$$
D \gg 1 .
$$

Evidently this is not the case for the frog myelinated nerve because $D=1$ by definition, but it is convenient to have an explicit expression for the wave speed in the continuum limit as a benchmark for numerical calculations. In the continuum limit, the wave speed $u \rightarrow u_{\mathrm{c}}$, where

$$
u_{\mathrm{c}}=\left(\frac{1-2 a}{\sqrt{2(1-a)}}\right) \sqrt{\frac{G}{R C^{2}}} \text { nodes/second } .
$$

To get the corresponding impulse speed in (say) meters/second, one multiplies this expression by $s$ in meters.

\section{Saltatory limit}

For $D$ of the order of unity or less, the wave of excitation jumps from node to node in a discontinuous matter, allowing the speed of conduction to be greatly increased without a corresponding increase in fiber diameter [41]. Since $D=1$ for the frog node, this myelinated nerve fiber lies within the saltatory range.

An additional feature of the saltatory limit is the possibility that the switching of one node is unable to bring the adjacent node above its threshold. In this situation - which is called failure - the impulse ceases to propagate $[1,4,5$, $10,11,23,25]$. Since failure of impulse conduction is an undesirable property of a real nerve, we expect the node spacing for a frog nerve to lie comfortably beyond this range.

If the internode spacing $s$ is increased so that $D$ is reduced to a critical value $D^{*}$, failure of impulse propagation occurs. Erneux and Nicolis have shown that this critical value of the discreteness parameter is given to lowest order in $a$ by [10]

$$
D^{*} \approx \frac{\beta a^{2}}{4},
$$

for the cubic form on the sodium conductance indicated in Equation (3). For $D$ slightly larger than $D^{*}$, these same authors show that the impulse velocity $u \rightarrow u_{\mathrm{s}}$, where 


$$
u_{\mathrm{s}}=\frac{\sqrt{\beta}}{\pi R_{\mathrm{f}} C} \sqrt{D-D^{*}} \text { nodes } / \text { second } .
$$

\section{Some numerical results and biological observations}

Equation (5) has been used to compute the wave front velocity for a nerve impulse, which is plotted against the discreteness parameter $(D)$ in Figure 2. From this plot, we draw the following conclusions.

- At larger values of the discreteness parameter $(D>5)$, the continuum approximation holds and impulse velocity is given by Equation (8).

- Failure in the model is accurately predicted by Equation (9) to occur at $D^{*}=0.2$.

- Near the failure point, impulse velocity is rather well represented by Equation (10).

- At $D=1$ (corresponding to a real frog nerve), neither the continuum approximation formula nor Equation (10) gives a satisfactory prediction of the impulse velocity.

In the context of these numerical results, we make the following empirical observations.

- Tasaki has reported measurements of the conduction velocities of 49 frog axons with diameters ranging from 4 to 16 microns at $24^{\circ} \mathrm{C}$ [43]. These data indicate that the experimental values of velocity $\left(u_{e}\right)$ are related to the outside diameter of the axon $(d)$ by the linear relationship

$$
u_{e}=2.5 d \pm 40 \% \text {, }
$$

where velocity is in meters per second and the diameter is in microns. The rather large error bars reflect the experimental difficulties involved in single axon measurements

From Figure 2, our calculated value for the impulse velocity on a single axon with a diameter of 14 microns is 29 meters per second, a value that lies within the bounds of Tasaki's empirical results.

- It might be objected that our choice of a cubic polynomial to represent sodium ion current in Equation (3) is not realistic, and we agree. The cubic polynomial is used because it allows us to make contact with the limiting forms of the continuum and saltatory limits in Equations (8) and (10) respectively. We have repeated our calculations of the impulse 


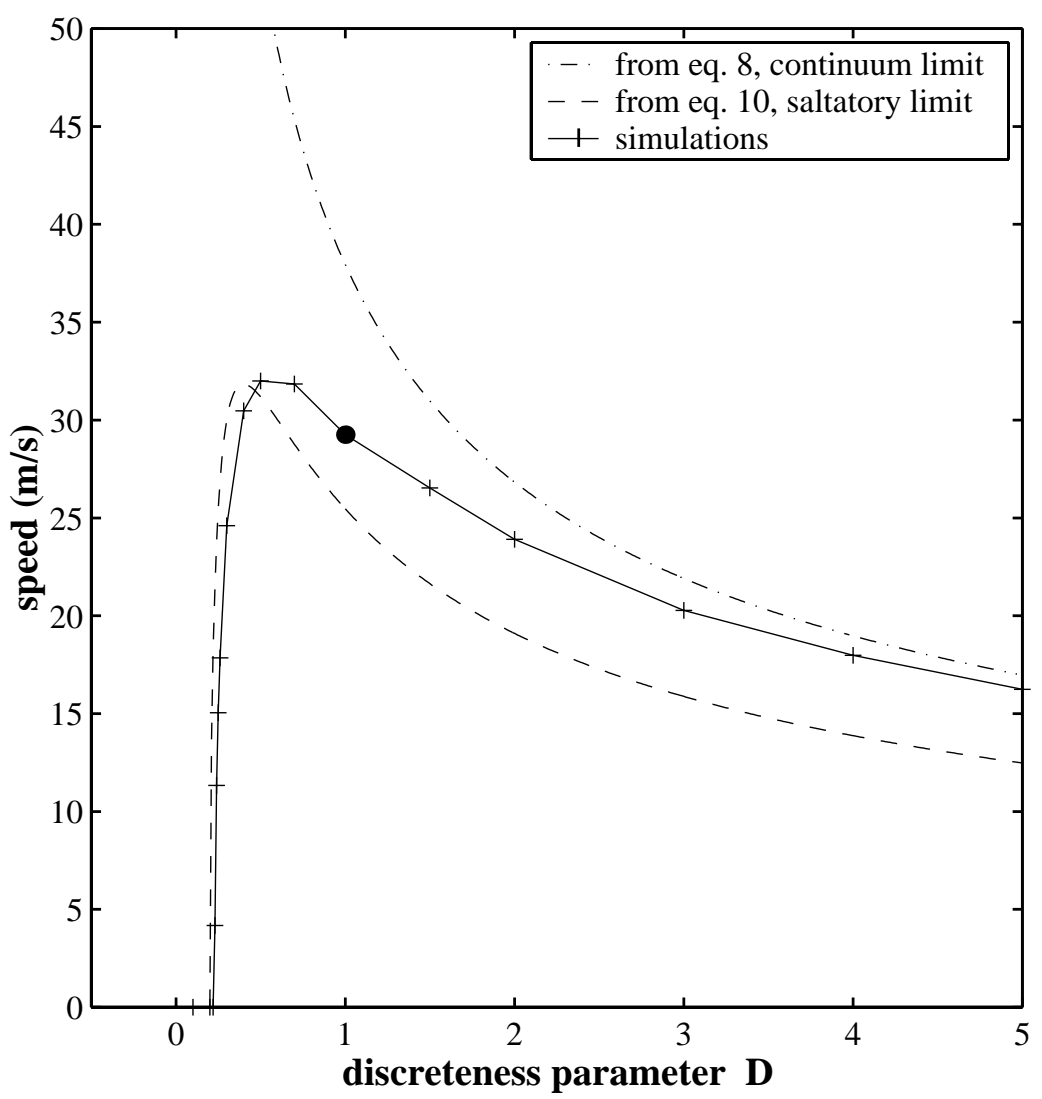

Figure 2: Leading edge impulse velocity on a myelinated axon as a function of the discreteness parameter $D$. The dashed line (- - - ) indicates the saltatory limit in Equation (10). The dot-dashed line (- . - . - - ) indicates the continuum limit of Equation (8). The "+" marks indicate numerical calculations. 
velocity with a Hodgkin-Huxley form for the sodium ion current that was adjusted to match experimental measurements of current at a frog node [8]. The resulting value of 46 meters per second also lies within the limits of Equation (11).

- Failure of an impulse is expected to occur at an internode spacing of 1 $\mathrm{cm}$, corresponding to $D=0.2$, whereas the normal nerve is designed for $D=1$, corresponding to a spacing of $.2 \mathrm{~cm}$. Thus the design of the axon provides a comfortable margin of safety against accidental failure on the frog's motor nerve.

- At $D=1$, Figure 2 shows that the impulse velocity of a normal frog nerve is close to the maximum possible value, suggesting an optimal design.

- Near $D=1$, the impulse velocity reaches its maximum value. This observation provides an explanation for the "surprising" fact - noted by Moore et al. [31] - that the conduction velocity of a frog's myelinated motor nerve is rather insensitive to the internode spacing. From an engineering perspective, operating in this insensitive region of parameter space makes the system more robust.

\section{Theoretical studies of ephaptic coupling}

In the parallel nerve interactions observed experimentally in the early 1940s by Katz and Schmitt [22] and by Arvanitaki [2], the mechanism of impulse coupling was through an electrical linking of the external return currents of nearby impulses.

An analytic formulation of such an interaction between nonmyelinated impulses was presented by Markin in 1970 [29, 30, 40] and experimentally verified by Ramón and Moore in 1978 [35]. This coupling effect occurs because the external current loop (from leading to trailing edge) of one impulse induces a longitudinal voltage in the external resistance, influencing the dynamics of an adjacent impulse. As it turns out, it is the second spatial derivative of the impulse on one fiber that perturbs the other impulse, and vice-versa [40]. (We note that this ephaptic interaction differs from the ohmic interaction through "gap junctions" to neighboring cells that has been considered by some other authors $[6,7,24]$.

\subsection{The continuum limit}

One effect of the mutual interaction between two nonmyelinated impulses is to cause them to propagate with exactly the same speed, an effect that was studied using a perturbation theory in the late 1970s [9, 41, 42]. In this analysis the 
small parameter is the mutual coupling between the two fibers, which can be expressed as the ratio

$$
\alpha \equiv R_{\mathrm{o}} /\left(R_{\mathrm{i}}+R_{\mathrm{o}}\right),
$$

relating the (shared) external resistance to the total longitudinal resistance.

To appreciate this result, consider two nonmyelinated nerves (i.e., the continuum limit) for which the impulse on fiber 2 leads (is ahead of) the impulse on fiber 1 by a distance $\delta$ and denote the impulse velocity on fiber $1(2)$ as $u_{1}(\delta)$ $\left(u_{2}(\delta)\right)$. Following the analysis presented in Section 7.6.2 of reference [41], it is straightforward to show that for a general class of simple nonlinear diffusion equations

$$
u_{1}(\delta)-u_{2}(\delta)=\frac{\alpha}{N} \int_{-\infty}^{\infty} e^{u_{0} x} f^{\prime}(x)\left[f^{\prime \prime}(x-\delta)-f^{\prime \prime}(x+\delta)\right] d x,
$$

where

$$
N \equiv \int_{-\infty}^{\infty} e^{u_{0} x}\left[f^{\prime}(x)\right]^{2} d x
$$

For the cubic representation of the sodium ion conductance that we have assumed in Equation (3), $f(x)=1 /\left(1+e^{x / \sqrt{2}}\right)$ and $u_{0}=(1-2 a) / \sqrt{2}$, which are the shape of the leading edge of an impulse and its speed of conduction, given in Equation (7). In this case, Equation (13) becomes

$$
\begin{gathered}
u_{1}(\delta)-u_{2}(\delta)=\frac{3 \alpha}{\sqrt{2}}\left(\frac{\Delta}{a(1-a)(\Delta-1)^{3}}\right) \times \\
{\left[2 \frac{\Delta^{1-2 a}-\Delta^{1+2 a}}{\Delta-1}-\Delta-1+\frac{\left(\Delta^{1-2 a}-\Delta^{-1+2 a}\right)\left(2(\Delta-1)^{2} a^{2}+\Delta(4 a+1)\right)}{(\Delta-1)(1-2 a)}\right]}
\end{gathered}
$$

where $\Delta=\exp (\delta / \sqrt{2})$.

From Equation (13), it can be seen that $\left[u_{1}(\delta)-u_{2}(\delta)\right]$ is a function of $\delta$ that goes through the origin with positive slope. Since

$$
\frac{d \delta}{d t}=-\left[u_{1}(\delta)-u_{2}(\delta)\right]
$$

$\delta$ decays to zero, indicating a stable locking of pairs of impulses at $\delta=0$. In other words, if impulse 2 gets ahead of impulse 1 , then impulse 1 will go faster than impulse 2 , thereby closing the gap. 


\subsection{The saltatory limit}

Moving slightly away from the continuum limit, the analysis upon which Equation (13) is based can be repeated with $x$-derivatives interpreted as differences and inner products written as sums; thus for $1 \ll D<\infty$, the behavior of ephaptically coupled myelinated nerves should be similar to that of nonmyelinated nerves. As $D$ is reduced toward the saltatory realm, however, the corresponding analysis becomes qualitatively different; a key question being: How long does it take the $(n+1)$ th node to reach a threshold level for ignition after the $n$th node fires?

An approximate answer to this question is provided by assuming that each node switches from 0 to $V_{\mathrm{b}}$ as a Heaviside step function, causing the voltage across the next node to rise as [41]

$$
V \sim \frac{V_{\mathrm{b}}}{1+R G_{0}}\left(1-e^{-t / \tau}\right),
$$

where $G_{0} \equiv V_{\mathrm{a}} G /\left(V_{\mathrm{b}}-V_{\mathrm{a}}\right)$ is the ionic conductance near zero voltage and

$$
\tau=R C /\left(1+R G_{0}\right)
$$

is the relevant time constant for charging the node capacitance. (In these expressions, we recall that $R=28$ megohms, and note that $1 / G_{0}=7.7$ megohms, so $1+R G_{0}=4.6$.)

As the voltage across the next node capacitance rises, the current that flows through the myelin sheath and returns through the external space relaxes with the same time constant from $V_{\mathrm{b}} / R$ to $V_{\mathrm{b}} G_{0} /\left(1+R G_{0}\right)$, a drop of about $20 \%$. With these facts in hand, we are in a position to understand why coupled impulses are also expected in the saltatory limit.

Assuming two parallel fibers with active nodes aligned and sharing an external resistance $R_{\mathrm{o}}$, the logic proceeds as follows.

- If impulses on the two fibers are exactly synchronized so that nodes $n$ switch at time zero, the currents flowing through $R_{\mathrm{o}}$ will tend to decrease the voltage across the $(n+1)$ th nodes. This has the effect of increasing the time needed to reach threshold at the $(n+1)$ th nodes, thereby decreasing the impulse velocity on both fibers.

- If the $n$th node on fiber 2 switches shortly before the corresponding node on fiber 1 (indicating that the impulse on fiber 2 leads the impulse on fiber 1 ), then the external return current from fiber 1 will be larger when the $(n+1)$ th node of fiber 2 is ready to fire, because the current from node $n$ to $(n+1)$ on fiber 1 has had less time to relax.

- Since the external current is greater when fiber 2 is ready to fire its subsequent node, the time for this firing will increase, implying that the impulse velocity on fiber 2 is diminished. 
- Similarly if the $n$th node on fiber 1 is assumed to switch slightly after that of fiber 2 , the external return current from fiber 2 will be smaller when the $(n+1)$ th node of fiber 1 is ready to fire, because the current from node $n$ to $(n+1)$ on fiber 2 has had more time to relax.

- Since the external current is less when fiber 1 is ready to fire its subsequent node, the time for this firing will decrease, implying that the impulse velocity on fiber 1 is increased.

- Thus the assumption that an impulse on fiber 2 leads an impulse on fiber 1 implies that the speed on the impulse on fiber 2 (fiber 1) decreases (increases), thereby closing the gap.

In the following section, detailed numerical studies are used to confirm this qualitative argument and explore the properties of ephaptically coupled myelinated nerves in the parameter range between the continuum and the saltatory limits.

\section{Numerical observations of ephaptic coupling}

In describing the interaction between myelinated nerve fibers, it is necessary to consider the degree to which the locations of the active nodes are aligned. To this end, we sketch two partially aligned axons in Figure 3(a) and a corresponding circuit diagram in Figure 3(b).

In this circuit diagram, the $V_{n}^{(j)}$ are voltages across the active nodes, where $j=1,2$ indicates a particular fiber. Similarly, the $I_{n}^{(j)}$ are mesh currents, which provide the independent variables for an analysis in terms of Kirchhoff's voltage law. Equating all of the voltages about the meshes to zero leads directly to the equations

$$
\begin{aligned}
& V_{n}^{(1)}-V_{n+1}^{(1)}=\left(R_{\mathrm{i}}+R_{\mathrm{o}}\right) I_{n}^{(1)}+R_{\mathrm{o}}\left[A I_{n}^{(2)}+(1-A) I_{n-1}^{(2)}\right] \\
& V_{n}^{(2)}-V_{n+1}^{(2)}=\left(R_{\mathrm{i}}+R_{\mathrm{o}}\right) I_{n}^{(2)}+R_{\mathrm{o}}\left[A I_{n}^{(1)}+(1-A) I_{n+1}^{(1)}\right],
\end{aligned}
$$

and the voltages across the active nodes are then related to the mesh currents by

$$
I_{n-1}^{(j)}-I_{n}^{(j)}=C \frac{d V_{n}^{(j)}}{d t}+I_{\mathrm{ion}, n}^{(j)}
$$

with

$$
I_{\mathrm{ion}, n}^{(j)}=\left(\frac{G}{V_{\mathrm{b}}\left(V_{\mathrm{b}}-V_{\mathrm{a}}\right)}\right) V_{n}^{(j)}\left(V_{n}^{(j)}-V_{\mathrm{a}}\right)\left(V_{n}^{(j)}-V_{\mathrm{b}}\right) .
$$



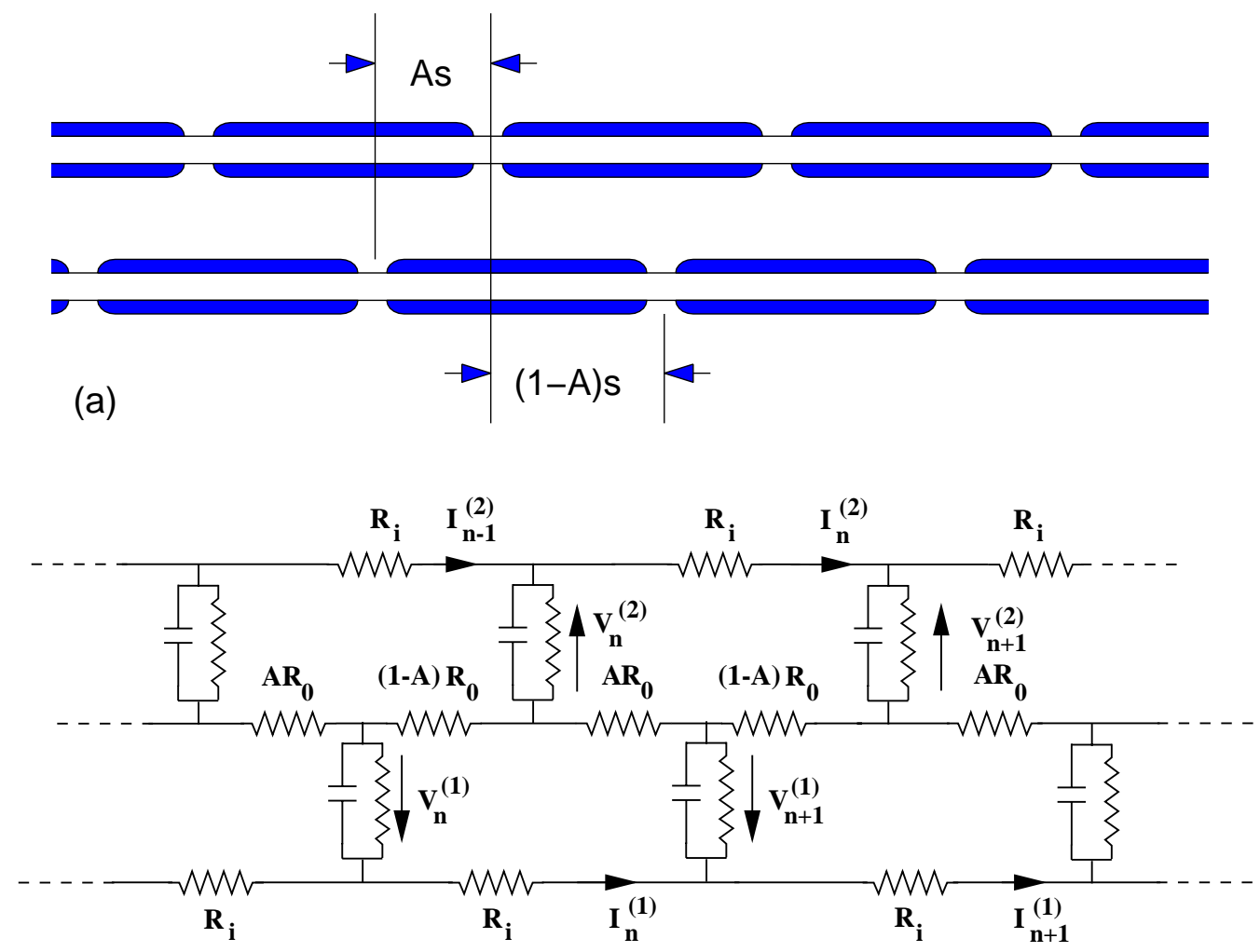

(b)

Figure 3: (a) Two myelinated nerves that are coupled by a linking (ephaptic coupling) of their external return currents. (Not to scale.) (b) A circuit diagram of the coupled myelinated nerves. 
In this formulation, an alignment parameter $A$ is introduced to indicate the degree of nodal alignment. With $A=1$, the active nodes on the two fibers are exactly aligned, whereas they are evenly staggered for $A=1 / 2$.

In Equations (16) and (17), it is convenient to measure voltages and currents in units of $V_{\mathrm{b}}$ and $V_{\mathrm{b}} / R_{\mathrm{f}}$ respectively. Thus the general equations for ephaptic coupling become

$$
\begin{gathered}
D\left(v_{n}^{(1)}-v_{n+1}^{(1)}\right)=i_{n}^{(1)}+\alpha\left[A i_{n}^{(2)}+(1-A) i_{n-1}^{(2)}\right] \\
D\left(v_{n}^{(2)}-v_{n+1}^{(2)}\right)=i_{n}^{(1)}+\alpha\left[A i_{n}^{(1)}+(1-A) i_{n+1}^{(1)}\right] \\
i_{n-1}^{(j)}-i_{n}^{(j)}=R_{\mathrm{f}} C \frac{d v_{n}^{(j)}}{d t}+i_{\text {ion }, n}^{(j)} \\
i_{\text {ion }, n}^{(j)}=\beta v_{n}^{(i)}\left(v_{n}^{(j)}-a\right)\left(v_{n}^{(j)}-1\right),
\end{gathered}
$$

where $v_{n}^{(j)} \equiv V_{n}^{(j)} / V_{\mathrm{b}}, i_{n}^{(j)} \equiv R_{f} I_{n}^{(j)} / V_{\mathrm{b}}, \beta \equiv R_{f} G /(1-a), R \equiv\left(R_{\mathrm{i}}+R_{\mathrm{o}}\right)$, $\alpha \equiv R_{\mathrm{o}} / R, D \equiv R_{\mathrm{f}} / R$, and $j=1,2$.

For $1 / 2<A<1$, it is difficult to further simplify these equations, but for $A=1$ and with the notation

$$
\Delta v^{(j)} \equiv\left(v_{n+1}^{(j)}-2 v_{n}^{(j)}+v_{n-1}^{(j)}\right),
$$

Equations (18) can be written to first order in $\alpha$ as

$$
\begin{aligned}
& D\left[(1-\alpha) \Delta v^{(1)}-\alpha \Delta v^{(2)}\right]=R_{\mathrm{f}} C \frac{d v_{n}^{(1)}}{d t}+\beta v_{n}^{(1)}\left(v_{n}^{(1)}-a\right)\left(v_{n}^{(1)}-1\right) \\
& D\left[(1-\alpha) \Delta v^{(2)}-\alpha \Delta v^{(1)}\right]=R_{\mathrm{f}} C \frac{d v_{n}^{(2)}}{d t}+\beta v_{n}^{(2)}\left(v_{n}^{(2)}-a\right)\left(v_{n}^{(2)}-1\right) .
\end{aligned}
$$

\subsection{Speed of coupled impulses}

In Figure 4, we show how the speed of two coupled impulses depends upon the ephaptic coupling constant $\alpha$. (Note that the curve for $\alpha=0$ is identical to that of Figure 2.) In Figure 4, three cases are of particular interest.

- The continuum limit

For $D \gg 1$ and $A=1$, we can assume that $\delta=0$ and all voltages and currents are identical on the two fibers, whereupon it is straightforward to show that the speed of two coupled impulses is decreased by a factor of $\sqrt{1+\alpha}$, which is observed numerically. For $A=1 / 2$, we also find that the impulse velocity is decreased by a factor of $\sqrt{1+\alpha}$ in the continuum limit. This is to be expected because the average values of the currents and voltages over several nodes is independent of node alignment in the continuum limit. 

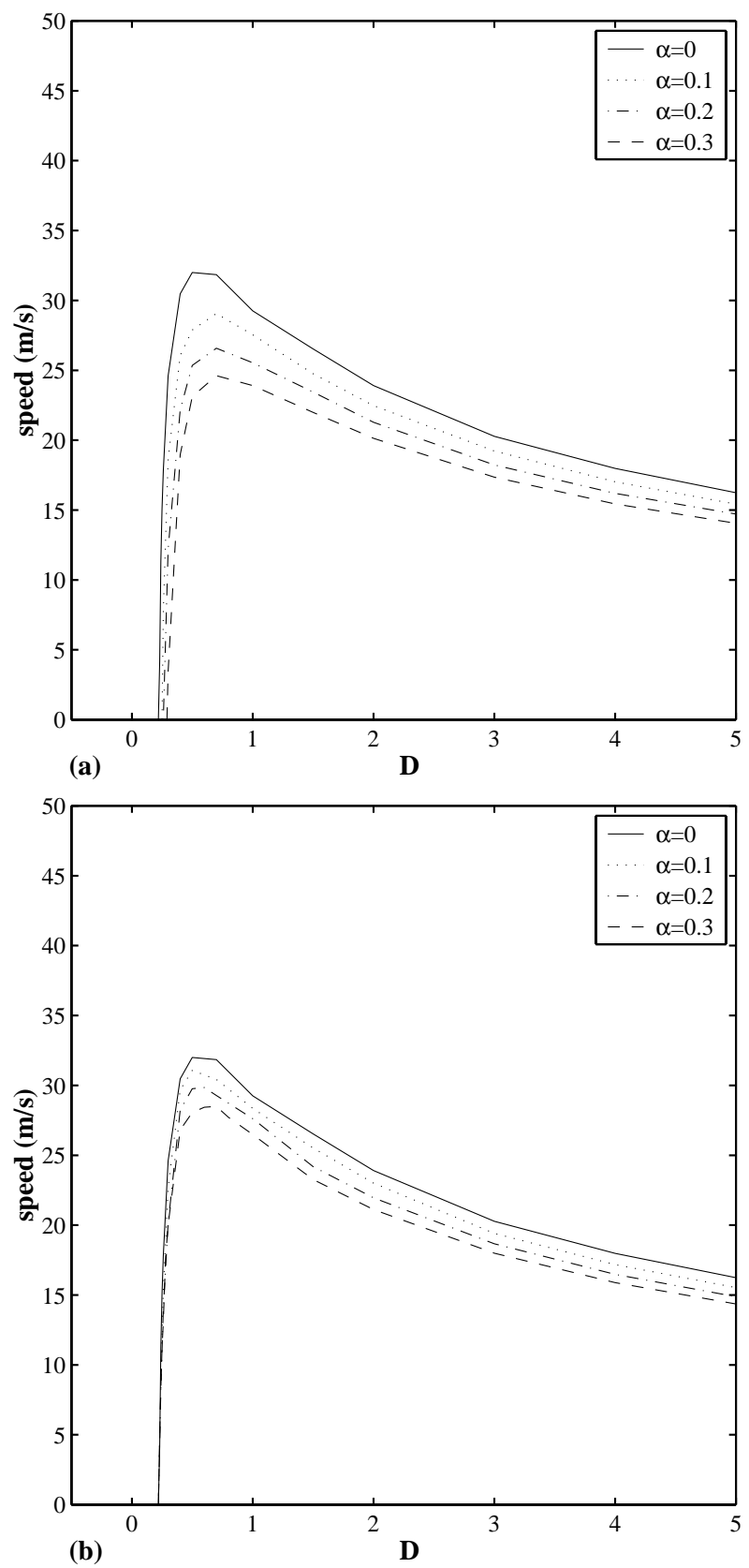

Figure 4: Coupled impulse speed as a function of coupling constant $(\alpha)$ and discreteness parameter $(D)$. (a) $A=1$. (b) $A=1 / 2$. 


\section{- Failure}

For $A=1$ and $\delta=0$ (two coupled impulses), twice the external current flows through the external resistance $\left(R_{\mathrm{o}}\right)$; thus the effective loop resistance seen be each fiber is

$$
R_{\mathrm{i}}+2 R_{\mathrm{o}}=R(1+\alpha) .
$$

Noting from Equation (9) that $D^{*}$ is proportional to $\beta$, which in turn is proportional to the effective loop resistance, we expect to find

$$
\frac{D^{*}(\alpha)}{D^{*}(0)}=1+\alpha,
$$

and this is confirmed by the numerical plot of Figure 5. More generally, Equation (10) implies that

$$
u_{\mathrm{s}}=\frac{\sqrt{\beta}}{\pi R_{f} C}\left(\frac{D}{\sqrt{1+\alpha}}-\frac{\beta a^{2}}{4} \sqrt{1+\alpha}\right) \text { nodes per second }
$$

as $D \rightarrow D^{*}$, which is confirmed by our numerical computations in Figure 4.

For $A=1 / 2$, the dependence of $D^{*}$ on $\alpha$ is more intricate. Referring to Figure 3, we see that the nodes are evenly staggered for $A=1 / 2$, implying an external resistance of $R_{\mathrm{o}} / 2$ linking adjacent current loops. The numerical studies show that in the saltatory limit the jumps alternate, first on one fiber and then on the other. Thus the effective loop resistance can be computed for a single fiber as

$$
R_{\mathrm{i}}+2\left(\frac{R_{\mathrm{o}}}{2}\right) \|\left(R_{\mathrm{i}}+R_{\mathrm{o}} / 2\right)
$$

where the symbol "||" implies evaluating the parallel combination of the resistors indicated, and contributions of order $R_{\mathrm{o}}^{3}$ have been neglected. Thus the effective loop resistance in this case is

$$
R_{\mathrm{i}}+R_{\mathrm{o}}-\frac{1}{2} \frac{R_{\mathrm{o}}^{2}}{R_{\mathrm{i}}+R_{\mathrm{o}}},
$$

which implies that

$$
\frac{D^{*}(\alpha)}{D^{*}(0)}=1-\frac{1}{2} \alpha^{2}+\mathrm{O}\left(\alpha^{3}\right) .
$$

Again, this is confirmed by the numerical results of Figure 5 . 


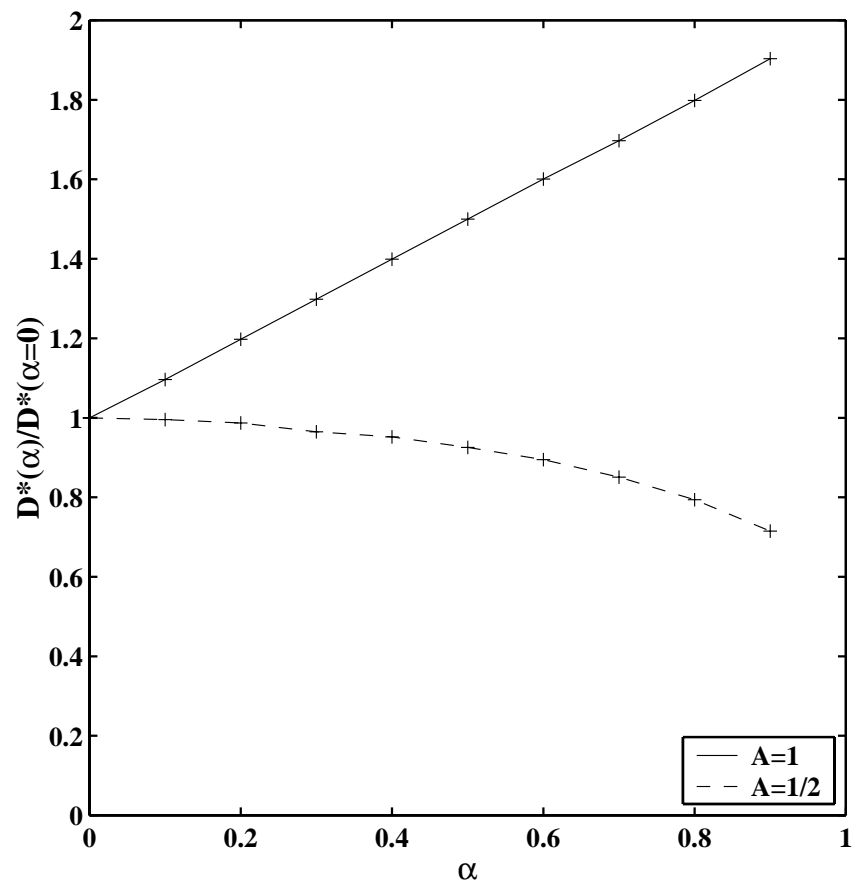

Figure 5: The value of the discreteness constant at which failure occurs $\left(D^{*}\right)$ as functions of $\alpha$ for $A=1$ and $A=1 / 2$. 
- Impulse velocity on a nerve bundle

From Figure 4 it is seen that the speed of locked impulses on two coupled fibers decreases as $\alpha$ is increased. For $n$ coupled fibers, the effective value of $\alpha$ is [30]

$$
\tilde{\alpha}=\frac{(n-1) R_{\mathrm{O}}}{R_{\mathrm{i}}+R_{\mathrm{o}}} .
$$

Assuming the fibers in a nerve bundle to be hexagonally arranged implies an $\tilde{\alpha}$ of $6 R_{\mathrm{o}} /\left(R_{\mathrm{i}}+R_{\mathrm{o}}\right)$, which might lead to an observable decrease in the speed of an individual axon.

\subsection{Dynamics of impulse coupling}

With reference to Equation (15), we recall that the dynamics of coupled impulses are governed by the function $\left[u_{1}(\delta)-u_{2}(\delta)\right]$, which is expected to be linearly dependent on $\alpha$ for sufficiently small values of $\alpha$.

From numerical integration of Equations (18), we have determined $d \delta / d t$ as a function of $\delta$, allowing construction of the three dimensional plots of

$$
\frac{u_{1}(\delta)-u_{2}(\delta)}{\alpha}
$$

as functions of $D$ and $\delta$ for $A=1$ and $A=1 / 2$ that are presented in Figure 6 . In the continuum limit $(D \gg 1)$, as expected, these curves approach the theoretical expression of Equation (14), which was obtained from perturbation theory.

As $D$ becomes smaller, nearing the saltatory limit, we find that an alignment of the active nodes $(A=1)$ leads to a stronger and more localized synchronization of impulses than for the staggered case $(A=1 / 2)$. From a qualitative perspective this seems reasonable because it is the external resistance shared by two fibers that leads to impulse synchronization, and with $A=1$ this shared external resistance is situated between two nodes, whereas with $A=1 / 2$ it is between three nodes.

The biological significance of coupled impulses may arise from at least three considerations [42].

- Synchronization on bundles of motor neurons might adjust the timings among coupled impulses, allowing for coordinated stimulation of muscle cells.

- Synchronization on bundles of optical or auditory axons in the central nervous system may insure the timings necessary for computations in the dendritic fields of subsequent neurons [41]. 
- Synchronization of groups of adjacent neurons in the corpus callosum (providing channels of communication between the two hemispheres of the brain) could be a means for the transmission of more intricate messages between the two hemispheres of the brain.

With such speculations in mind, it is interesting to consider whether an observation of aligned nodes should be taken to imply the functional importance of coupled impulses. (This is more than a hypothetical question, because nodal alignment of small groups of fibers has been observed in the corpus callosum [45].) Although it might be expected from a qualitative perspective, our data suggest that this question be approached with caution, for the following reasons. (i) While the tendency to synchronize is stronger for $A=1$, it is spread out over two internodes for $A=1 / 2$, indicating a more robust coupling, so both of these limiting cases lead to impulse synchronization at $\delta=0$. Thus if the fibers are short (with few nodes), the need for rapid synchronization may require node alignment, whereas rapid synchronization would be less important for longer fibers. (ii) The node separation at which failure occurs is insensitive to ephaptic coupling for staggered nodes, whereas it decreases linearly with the coupling for aligned nodes.

In any case, Figure 6 indicates that ephaptic coupling is to be expected on myelinated fibers for every sort of nodal orientation: aligned, unaligned, or random.

\section{Conclusions}

For an isolated myelinated nerve axon, we have confirmed previous calculations of impulse speeds in the continuum and saltatory limits, showing that conduction velocity lies between these two limits, near its maximum value.

Taking ephaptic coupling of parallel fibers into consideration, we have obtained the following results. (i) Estimates of the effects of impulse coupling in the saltatory and continuum limits are confirmed and extended to the intermediate realm. (ii) The effects of impulse coupling on failure are computed and found in agreement with theoretical estimates in the saltatory limit. (iii) The decrease of impulse velocity in motor nerves due to impulse coupling is calculated and found to be significant. (iv) In the saltatory range, we show that the coupling of impulses is to be expected for any degree of nodal alignment.

\section{Acknowledgments}

We thank Stephen G. Waxman for helpful comments on the alignment of active nodes on myelinated fibers, and James P. Keener for his careful review of the manuscript, which led to several improvements. Also we appreciate support for several visits among Dijon, Edinburgh and Lyngby - by the European Commission's Training and Mobility of Researchers (TMR) Programme under the 


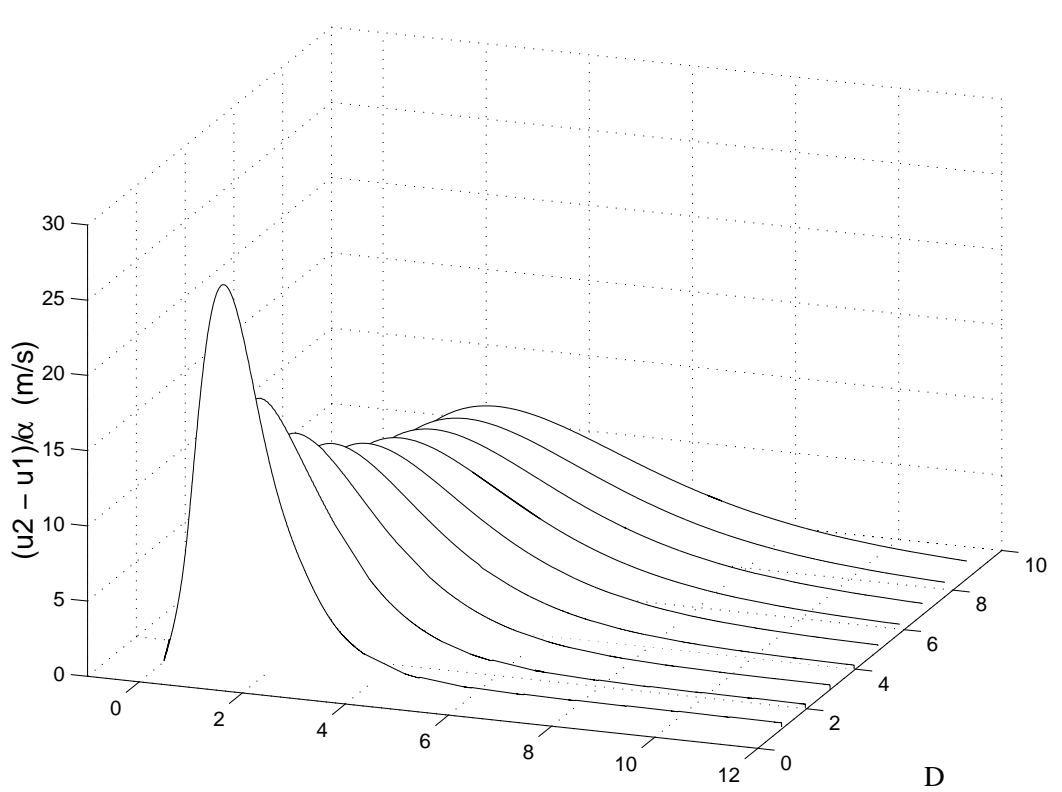

(a)

$\delta$ (nodes)

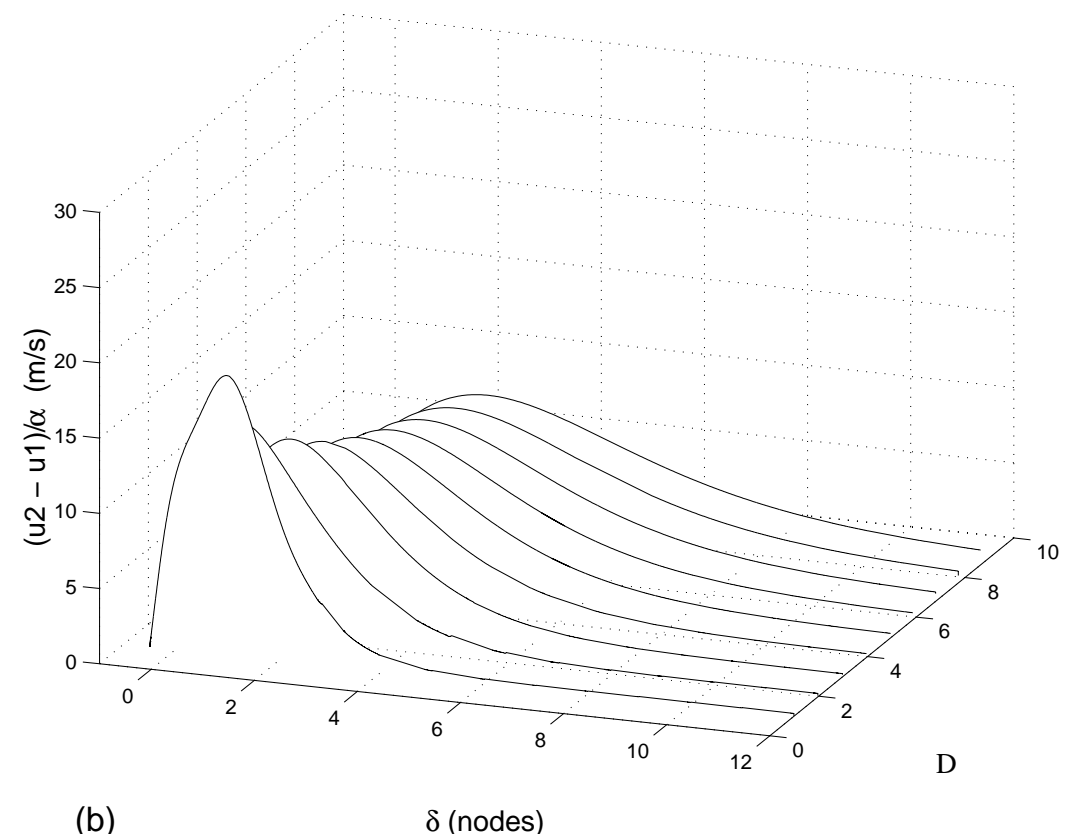

Figure 6: Relative speeds of two impulses $\left(u_{1}-u_{2}\right) / \alpha$ as functions of both discreteness parameter $(D)$ and impulse spacing $(\delta)$. (a) $A=1$. (b) $A=1 / 2$. 
grant ERBFMGECT950051, the Danish Research Academy, and Heriot-Watt University - which have facilitated the progress of this research.

\section{Appendix - Numerical details}

The numerical simulations of the ephaptic coupling are performed using a predictorcorrector algorithm in which each of the two fibers is made up of $N=200$ cells. The predictor step consists of applying the simple Euler predictor to Equation (17), then solving the algebraic Equations (16). The corrector step follows the same strategy, using the trapezoidal rule corrector for Equation (17).

The initial conditions are set as step functions, which evolve into propagating topological waves.

The next step is to measure the relative speed of two impulses $\left(u_{1}-u_{2}\right) / \alpha$ as functions of both discreteness parameter $D$ and impulse spacing $\delta$. We define the position of each wave as the point at which it reaches a particular value of $V_{c}$. This value is chosen to half of the maximum amplitude, i. e. $V_{c}=1 / 2$. The corresponding point on the $n$ axis, $N_{c}$ is found by calculating a linear interpolant to $V_{n}$ using the values on either side of of $V_{c}$, then solving for $V_{n}=V_{c}$.

This local linear approximation to the profile generates some small high frequency errors which are removed using a low pass filter, namely a Butterworth filter of the third order.

The relative speed $\left(u_{1}-u_{2}\right)$ is then determined as a numerical derivative of the distance $\left(N_{c 1}-N_{c 2}\right)$ between the adjacent waves, leading to the results plotted on figure 6 .

\section{References}

[1] A. R. A. Anderson and B. D. Sleeman, Wave front propagation and failure in coupled systems of discrete bistable cells modeled by FitzHugh-Nagumo dynamics, Int. J. of Bifurcation and Chaos 5 (1995) 63-74.

[2] A. Arvanitaki, Effects evoked in an axon by the activity of a contiguous one, J. Neurophysics 5 (1942) 89-108.

[3] M. H. Brill, S. G Waxman, J. W Moore and R. W Joyner, Conduction velocity and spike configuration in myelinated fibers, J. Neurol. Neurosurg. Psychiatry 40 (1977) 769-774.

[4] V. Booth and T. Erneux, Mechanisms for propagation failure in discrete reaction-diffusion systems, Physica A 188 (1992) 206-209.

[5] V. Booth and T. Erneux, Understanding propagation failure as a slow capture near a limit point, SIAM J. Appl. Math. 55 (1995) 1372-1389. 
[6] A. Bose, Symmetric and antisymmetric pulses in parallel coupled nerve fibers, SIAM J. Appl. Math. 55 (1995) 1650-1674.

[7] A. Bose and C. K. R. T. Jones, Stability of the in-phase travelling wave solution in a pair of coupled nerve fibers, Indiana Univ. Math. J. 44 (1995) $189-220$.

[8] K. S. Cole, Membranes, Ions and Impulses, Univ. of California Press, Berkeley, 1968.

[9] J. C. Eilbeck, S. D. Luzader and A. C. Scott, Pulse evolution on coupled fibers, Bull. Math. Biol. 43 (1981) 389-400.

[10] T. Erneux and G. Nicolis, Propagating waves in discrete bistable reactiondiffusion systems, Physica D 67 (1993) 237-244.

[11] G. Fáth, Propagation failure of traveling waves in a discrete bistable medium, Physica D 116 (1998) 176-180.

[12] R. FitzHugh, Impulses and physiological states in theoretical models of nerve membrane, Biophys. J. 1 (1961) 445-466.

[13] R. FitzHugh, Computation of impulse initiation and saltatory conduction in a myelinated nerve fiber, Biophys. J. 2 (1962) 11-21.

[14] B. Frankenhaeuser and A. F. Huxley, The action potential in the myelinated nerve fiber of Xenopus laevis as computed on the basis of voltage clamp data, J. Physiol. (London) 171 (1964) 302-325.

[15] L. Goldman and J. S. Albus, Computation of impulse conduction in myelinated fibers, Biophys. J. 8 (1968) 596-607.

[16] I. S. Gradshteyn and I. M. Ryzhik, Tables of Integrals, Series, and Products, Academic Press, San Diego, 1980.

[17] A. L. Hodgkin and A. F. Huxley, A quantitative description of membrane current and its application to conduction and excitation in nerve, J. Physiol. 117 (1952) 500-544.

[18] A. L. Hodgkin, The Conduction of the Nervous Impulse, Liverpool University Press, Liverpool, 1964.

[19] N. A. Hutchinson, Z. J. Koles and R. S. Smith, Conduction velocity in myelinated nerve fibers of Xenopus laevis, J. Physiol. 208 (1970) 279-289.

[20] J. J. B. Jack, D. Noble and R. W. Tsien, Electric Current Flow in Excitable Cells, Oxford University Press, Oxford, 1975. 
[21] J. G. R. Jefferys, Nonsynaptic modulation of neuronal activity in the brain: Electric currents and extracellular ions, Physiol. Rev. 75 (1995) 689-723.

[22] B. Katz and O. H. Schmitt, Electric interaction between two adjacent nerve fibers, J. Physiol. 97 (1940) 471-488.

[23] J. P. Keener, Propagation and its failure in coupled systems of discrete excitable cells, SIAM J. Appl. Math. 47 (1987) 556-572.

[24] J. P. Keener, Frequency decoupling of parallel excitable fibers, SIAM J. Appl. Math. 49 (1989) 211-230.

[25] J. Keener and J. Sneyd, Mathematical Physiology, Springer-Verlag, New York, 1998.

[26] H. Kunov, Controllable piecewise-linear lumped neuristor realization, Electron. Lett. 1 (1965) 134.

[27] H. Kunov, Nonlinear Transmission Lines Simulating Nerve Axon, PhD Thesis, Electronics Laboratory, Technical University of Denmark, 1966.

[28] H. Kunov, On recovery in a certain class of neuristors, Proc. IEEE 55 (1967) 427-428.

[29] V. S. Markin, Electrical interactions of parallel nonmyelinated fibers I. Change in excitability of the adjacent fiber, Biophysics, 15 (1970) 122-133.

[30] V. S. Markin, Electrical interactions of parallel nonmyelinated fibers II. Collective conduction of impulses, Biophysics, 15 (1970) 713-721.

[31] J. W. Moore, R. W. Joyner, M. H. Brill, S. G. Waxman and M Najar-Joa, Simulations of conduction in uniform myelinated fibers: Relative sensitivity to changes in nodal and internodal parameters, Biophys. J. 21 (1978) 147160.

[32] J. Nagumo, S. Arimoto, and S. Yoshizawa, An active impulse transmission line simulating nerve axon, Proc. IRE 50 (1962) 2061-2070.

[33] W. F. Pickard, On the propagation of the nervous impulse down medulated and unmedulated fibers, J. theor. Biol. 11 (1966) 30-45.

[34] W. F. Pickard, Estimating the velocity of propagation along myelinated and unmyelinated fibers, Math. Biosci. 5 (1969) 305-319.

[35] F. Ramón and J. W. Moore, Ephaptic transmission in squid giant axons, Am. J. Physiol. 234 (1978) C162-C169.

[36] I. Richer, Pulse transmission along certain lumped nonlinear transmission lines, Electron. Lett. 1 (1965) 135-136. 
[37] I. Richer, The switch-line: A simple lumped transmission line that can support unattenuated propagation, IEEE Trans. Circuit Theory CT-13 (1966) 388-392.

[38] W. A. H. Rushton, A theory of the effects of fibre size in medullated nerve, J. Physiol. (London) 115 (1951) 101-122.

[39] A. C. Scott, Neuristor propagation on a tunnel diode loaded transmission line, Proc. IEEE 51 (1963) 240.

[40] A. C. Scott, The electrophysics of a nerve fiber, Rev. Mod. Physics, 47 (1975) 487-533.

[41] A. C. Scott, Nonlinear Science: Emergence and Dynamics of Coherent Structures, Oxford University Press, Oxford, 1999.

[42] A. C. Scott and S. D. Luzader, Coupled solitary waves in neurophysics, Phys. Scr. 20 (1979) 395-401.

[43] I. Tasaki, Physiology and Electrochemistry of Nerve Fibers, Academic Press, New York, 1982.

[44] S. G. Waxman, J. D. Kocsis and P. K. Stys, The Axon: Structure, Function and Pathophysiology, Oxford University Press, New York, 1995.

[45] S. G. Waxman, private communication.

[46] J. Z. Young, Doubt and Certainty in Science, Oxford Clarendon Press, Oxford, 1951.

[47] Ya. B. Zel'dovich and D. A. Frank-Kamenetsky, K teorii ravnomernogo rasprostranenia plameni, Doklady Akademii Nauk SSSR, 19 (1938) 693697. 


\section{FIGURE CAPTIONS}

Figure 1. A single myelinated nerve fiber. (not to scale.)

Figure 2. Leading edge impulse velocity on a myelinated axon as a function of the discreteness parameter $D$. The dashed line (- - - ) indicates the saltatory limit in Equation (10). The dot-dashed line (- - - - - ) indicates the continuum limit of Equation (8). The "+" marks indicate numerical calculations.

Figure 3. (a) Two myelinated nerves that are coupled by a linking (ephaptic coupling) of their external return currents. (Not to scale.) (b) A circuit diagram of the coupled myelinated nerves.

Figure 4. Coupled impulse speed as a function of coupling constant $(\alpha)$ and discreteness parameter $(D)$. (a) $A=1$. (b) $A=1 / 2$.

Figure 5. The value of the discreteness constant at which failure occurs $\left(D^{*}\right)$ as functions of $\alpha$ for $A=1$ and $A=1 / 2$.

Figure 6. Relative speeds of two impulses $\left(u_{1}-u_{2}\right) / \alpha$ as functions of both discreteness parameter $(D)$ and impulse spacing $(\delta)$. (a) $A=1$. (b) $A=1 / 2$. 